\title{
Entre eurocentrisme et globalisation
}

Une lecture de « Globus »

\section{Francesco Paolo Ciglia}

\section{(2) OpenEdition}

\section{Journals}

Édition électronique

URL : https://journals.openedition.org/cps/2581

DOI : $10.4000 / c p s .2581$

ISSN : 2648-6334

Éditeur

Presses universitaires de Strasbourg

Édition imprimée

Date de publication : 1 juin 2011

Pagination : 53-65

ISBN : 978-2-354100-36-0

ISSN : $1254-5740$

Référence électronique

Francesco Paolo Ciglia, "Entre eurocentrisme et globalisation », Les Cahiers philosophiques de Strasbourg [En ligne], 29 | 2011, mis en ligne le 15 mai 2019, consulté le 11 novembre 2022. URL http://journals.openedition.org/cps/2581 ; DOI : https://doi.org/10.4000/cps.2581

\section{(c) (i) (2)(2)}

Creative Commons - Attribution - Pas d'Utilisation Commerciale - Partage dans les Mêmes Conditions 4.0 International - CC BY-NC-SA 4.0

https://creativecommons.org/licenses/by-nc-sa/4.0/ 


\section{Entre eurocentrisme et globalisation Une lecture de «Globus»}

Francesco Paolo Ciglia

\section{Un petit ouvrage qui donne à penser}

Dans cette contribution, je me propose essentiellement d'envisager d'une façon critique un petit ouvrage de Rosenzweig. L'ouvrage en question a été rédigé en 1917, donc, pendant la phase conclusive de la Première Guerre mondiale, lorsque son auteur faisait son service militaire sur le front des Balkans. Il s'intitule «Globus. Studien zur weltgeschichtlichen Raumlehre», c'est-à-dire «Le globe. Études sur la doctrine historico-universelle de l'espace $»^{1}$.

"Globus» appartient sans doute à la préhistoire de la pensée rosenzweigienne proprement dite. Il fut réalisé en effet, comme un produit provisoire, à la physionomie fort expérimentale, sous la poussée du tourbillon d'idées qui finira par aboutir, environ un an après, à la rédaction de L'Étoile de la Rédemption ${ }^{2}$, c'est-à-dire le chef-d'œuvre du penseur. C'est peut-être la raison pour laquelle son auteur, qui pourtant en avait une très grande considération, renonça finalement à le publier.

1 Louvrage a été publié après la mort de son auteur, dans: Rosenzweig (1984, p. 313-368). Le même ouvrage a été édité en français dans un volume qui contient aussi d'autres écrits rosenzweigiens, et qui s'intitule: Confluences. Politique, histoire, judaïsme, Rosenzweig (2003, p. 37-102). J'ai en fait une édition, en italien, sous forme d'un petit volume autonome, accompagné d'une introduction, de quelques documents épistolaires et d'un commentaire (Rosenzweig 2007). Les citations de "Globus» sont tirées de l'édition allemande citée.

2 Rosenzweig (1988); en français, Rosenzweig (1982). 
Le petit ouvrage est assurément très important, et pour différentes raisons, que je vais illustrer tout de suite.

$\mathrm{Au}$ point de vue strictement historique, le texte représente un document vraiment remarquable d'une phase intermédiaire de la démarche intellectuelle de son auteur. En 1912, Rosenzweig avait terminé sa thèse de Doctorat sur Hegel et l'État ${ }^{3}$. En 1913, il avait vécu une profonde crise existentielle et intellectuelle, à la suite de laquelle il avait pris désormais les décisions les plus importantes de sa vie. Après sa redécouverte tourmentée de son identité juive originaire, il s'était résolu, en effet, à refuser définitivement les perspectives de carrière universitaire que lui ouvraient ses études historiographiques hégéliennes, pour se vouer entièrement à la recherche spéculative personnelle et à la promotion des hautes études juives en Allemagne. L'ouvrage qui nous intéresse représente donc le premier témoignage littéraire d'une certaine consistance quantitative et qualitative de la nouvelle orientation qu'avait prise la recherche du jeune savant. L'historien, que Rosenzweig n'était désormais plus, trace, en effet, dans "Globus», le bilan de toute sa recherche précédente, et le philosophe, qu'il était en train de devenir, commence à y faire ses premiers pas. En utilisant une image métaphorique, on pourrait bien dire que "Globus» représente le lieu littéraire critique où le premier - c'est-à-dire l'historien qui trouverait son expression la plus pleine dans Hegel et l'État - passe idéalement le témoin au deuxième - c'est-à-dire au philosophe qui allait dire sa parole définitive dans L'Étoile de la Rédemption.

Mais la signification du petit ouvrage ne s'épuise certes pas au niveau historico-philologique. Il contient en effet bien plus, à savoir un message authentiquement spéculatif et d'une très grande suggestion, qui mérite d'être envisagé avec soin. Je vais essayer de le faire, bien que très rapidement.

\section{Une méditation sur l'histoire humaine}

"Globus» se présente essentiellement comme l'ébauche, très fascinante, d'une méditation globale sur l'histoire humaine universelle. Cette méditation est développée toutefois à partir d'un point de vue insolite ou même surprenant. La longue et lente démarche de

3 Rosenzweig, (1962); en français Rosenzweig (1991). 
l'histoire humaine universelle est comprise, dans l'ouvrage, comme une organisation et réorganisation toujours renouvelées des deux articulations fondamentales de la spatialité géographique, c'est-à-dire, la spatialité terrestre et la spatialité maritime. Rosenzweig s'efforce donc dans l'ouvrage de saisir en historien, à chaque époque, l'entrecroisement et l'interdépendance entre la temporalité historique et la spatialité géographique. La méditation géopolitique sur l'histoire universelle est toutefois contextualisée à l'intérieur d'un horizon tout à fait philosophique. "Globus» se présente donc comme la synthèse singulière d'une recherche historiographique très engagée et d'une philosophie de l'histoire en bonne et due forme.

Il est naturellement impossible d'examiner ici en détail la riche et complexe intrigue qui constitue la trame du petit ouvrage. Je me limiterai essentiellement à deux points fondamentaux. Je vais envisager en premier lieu l'idée de l'Europe - de son identité, de sa vicissitude historique et de son destin - que Rosenzweig nous propose dans son petit ouvrage. Deuxièmement, je vais examiner les pressentiments, très ponctuels et parfois même frappants, des phénomènes de la globalisation qui s'en dégagent. L'analyse de ces deux points devra toutefois être précédée nécessairement d'une reconstruction très rapide des lignes essentielles de la plus générale philosophie de l'histoire qui est contenue dans notre texte.

"Globus» débute par une méditation sur le premier commencement, la fin et la démarche globale de l'histoire humaine universelle. Cette démarche est amorcée d'emblée - et elle est ensuite même dominée et orientée -, selon Rosenzweig, par l'interaction entre deux forces motrices historiques antagoniques et toutefois complémentaires. L'histoire universelle commence en effet lorsque le premier homme découpe une partie du sol terrestre comme propriété pour soi et pour les siens. Cet acte crucial met en marche la dynamique inextinguible de la délimitation, de la séparation, du partage et de la différentiation réciproques entre les divers domaines du sol terrestre. C'est là la première force motrice qui pousse en avant l'histoire humaine. Le sol terrestre se couvre aussi d'un réseau épais de frontières, qui se déplacent et s'entrecroisent l'une l'autre sans cesse. Le long des lignes de ces frontières se développe un jeu relationnel très complexe entre les hommes. Ce jeu relationnel identifie, différencie, sépare et enfin relie le mien, le nôtre, le tien, le sien et le vôtre. Il me semble important de souligner ici que, pour Rosenzweig, 
au-dessous du réseau des frontières géo-historiques et politiques, il y a toujours l'homme et surtout les relations entre les hommes.

Avec la dynamique de la séparation, du partage et de la différentiation s'entrecroise toutefois la dynamique opposée de l'intégration réciproque progressive des différents domaines du monde. Cette deuxième force motrice de l'histoire universelle va conduire enfin, à travers un itinéraire très complexe, nullement linéaire et prédéterminé, jusqu’à une réunification finale et totale du globe terrestre. Mais c'est juste ici que devant les yeux du lecteur s'ouvre toute grande une puissante vision utopique et prophétique, qui présente des traits messianiques et eschatologiques. "Comme le premier Moi limité et son extension également limitée au premier Nous marque le premier instant de l'histoire", écrit l'auteur, «ainsi le dernier Nous illimité et son approfondissement également illimité au dernier Moi marque son dernier instant. Entre ce matin et ce soir se meuvent les frontières sur le sol et elles donnent une réponse à celui qui demande à quelle hauteur se place le soleil sur le ciel de l'histoire» (Rosenzweig 1984, p. 313). L'histoire humaine universelle trouve donc son premier commencement dans le contexte d'une terre unitaire, mais vide, indifférenciée, anonyme, c'est-à-dire dans le contexte d'un terrain abandonné. Cette terre unitaire, vide et indifférenciée se couvre progressivement des frontières qui la répartissent et l'articulent sans cesse. Mais cette terre partagée par des possesseurs divers, souvent en lutte entre eux, semble destinée à marcher vers une unité tout à fait différente par rapport à l'unité primitive. Il s'agit, en effet, d'une unité tissée d'un réseau de relations vivantes. Ces relations assument un visage humain. Elles vont composer enfin la figure d'une communauté universelle.

Les deux forces motrices de l'histoire universelle trouvent, selon Rosenzweig, aussi bien une métaphore particulièrement prégnante et suggestive que le lieu privilégié de leurs concrétisations respectives dans les deux articulations fondamentales de la spatialité que j’ai déjà évoquées auparavant. La spatialité terrestre semble en effet être marquée essentiellement par la possibilité constitutive d'être délimitée et répartie. Elle représente la métaphore et le lieu privilégié de la séparation, de la frontière et enfin de la finitude. La spatialité maritime au contraire semble marquée par l'impossibilité absolue d'être soit délimitée, soit répartie. Elle représente donc la métaphore et le lieu privilégié de l'unité sans limites et donc de l'infinitude. Les articulations fondamentales de 
la spatialité géographique ont toutefois toutes les deux une vocation et une destination très précises. Chacune d'entre elles est appelée en effet à soutenir, à l'intérieur de son propre horizon, l'accomplissement de l'œuvre caractéristique qui est promue par la force motrice de l'histoire universelle qui trouve son contexte privilégié dans l'horizon de l'autre articulation de la spatialité géographique. La destination ultime de la spatialité terrestre, par exemple, consiste dans l'intégration globale de tous ses domaines différenciés à l'intérieur d'une unité finale et sans limites. La destination ultime de la spatialité maritime au contraire consiste dans une différentiation interne bien articulée, jusqu'à ce que son unité originellement vide et stérile se transforme en une unité pleine, dynamique et vivante.

\section{L'histoire humaine entre Homère et la Bible}

La signification globale de la singulière intrigue qui se produit entre les articulations fondamentales de la spatialité géographique et les deux forces motrices de l'histoire universelle est naturellement susceptible d'interprétations les plus différentes. Dans son petit ouvrage Rosenzweig analyse seulement deux interprétations particulières - vraiment cruciales - de cette intrigue, qui résultent de deux images du monde qui ont été élaborées par l'Antiquité. Il s'agit des images du monde que Rosenzweig dénomme respectivement "homérique» et «biblique». Les deux adjectifs sont insérés toutefois à l'intérieur de prudents guillemets.

La représentation «homérique» du monde place au milieu du monde la grande masse d'une mer interne. Cette mer est renfermée tout autour par un anneau côtier vaste et bien articulé, qui est toutefois délimité à son tour par l'étroit ruban du fleuve mythique Océan. Dans cette image du monde essentiellement "méditerranéenne" c'est la spatialité terrestre qui joue le rôle prédominant, en dépit de l'évidence première et la plus immédiate. La terre ferme en effet délimite ou même endigue la spatialité maritime, de sorte que cette dernière est perçue comme le lieu qui rend possible la relation réciproque et la communication des différents domaines de la spatialité terrestre. La mer est donc, pour ainsi dire, mise au service de la terre ferme. Puisque la spatialité terrestre, comme l'on disait auparavant, semble être la métaphore et le lieu privilégié de la séparation et de la différence, l'image "homérique» 
du monde paraît dominée par conséquent par l'idée de la limite, de la finitude, de l'équilibre.

La représentation «biblique» du monde, comme on peut la trouver en particulier dans le deuxième Isaie, place au contraire au milieu du monde un bloc continental énorme, une terre ferme, "où les grands royaumes surgissent et passent» (Rosenzweig 1984, p. 349). Cette terre ferme est entourée par une gigantesque masse d'eau, qui ne peut pas être délimitée, à son tour, à l'intérieur de frontières bien tranchées. La spatialité maritime, qui joue dans l'image du monde "biblique» le rôle le plus important, assume de la sorte des traits abyssaux, océaniques. Elle suggère l'idée d'un éloignement extrême, d'un au-delà par rapport au monde délimité des hommes. Cette perception "océanique» de la mer est évidemment tout à fait différente par rapport à l'image "méditerranéenne» de la mer, que nous avons trouvée dans l'intuition «homérique» du monde. On pourrait dire que la représentation «biblique» du monde se fonde sur l'idée de l'inconnu, du mystère, de l'infinitude, qui tous marquent la condition humaine.

Nous avons déjà vu comment, pour Rosenzweig, aux deux modalités de la spatialité géographique, s'associent les deux forces motrices de l'histoire universelle. Il s'ensuit que, dans la perspective particulière de "Globus", l'image "homérique» du monde privilégierait les forces motrices historiques de la différentiation, tandis que l'image «biblicoprophétique» du monde privilégierait les forces motrices historiques de la réunification.

\section{Essence et idée de l'Europe}

Le contexte global de la philosophie de la géohistoire qui est développée dans "Globus", représente même l'horizon problématique général dans lequel est placée l'interrogation rosenzweigienne sur l'Europe. Cette interrogation se développe en un questionnement très original sur l'essence de l'Europe, sur son identité profonde, sur sa vocation la plus propre et enfin sur son destin ultime. L'interrogation en question n'est pas formulée certes, dans le petit ouvrage, d'une manière si explicite. Elle émerge toutefois toujours à nouveau, pour ainsi dire «entre les lignes» du texte, tout le long de "Globus». Rosenzweig répond en effet implicitement à cette interrogation lorsqu'il ébauche une figure tout à fait particulière de l'Europe dans son ouvrage. En 
résumant très brièvement un discours beaucoup plus long et complexe, on pourrait dire que l'Europe, comme idée et comme projet historicopolitique, est comprise essentiellement, dans "Globus», comme une synthèse d'importance fondamentale entre l'image "homérique" du monde et l'image "biblico-prophétique» de celui-ci, c'est-à-dire, comme une synthèse qui marque un véritable tournant historique planétaire. Cette représentation de l'Europe n'est certes pas nouvelle, puisqu'elle est attestée par une tradition historique très longue, richement modulée. Au premier abord, elle pourrait donc paraitre plutôt banale ou même usée. Dans le contexte très particulier de l'ouvrage qui nous intéresse cette vision de l'Europe assume toutefois une signification tout à fait spéciale et originale, qui doit être approfondie.

L'image «homérique» du monde souligne, d'une façon particulière, comme nous l'avons déjà dit, la spatialité terrestre. Cette image est donc placée par Rosenzweig sous le signe de la séparation, de la délimitation, de la finitude et de la différentiation. Mais la différentiation en question ne concerne pas seulement des domaines géographiques divers, qui sont situés l'un en face de l'autre ou l'un à côté de l'autre. Il s'agit même d'une différentiation qui subsiste entre des hommes, des peuples, des économies, des systèmes politiques, des langues et des cultures radicalement divers les uns par rapport aux autres. Le cœur palpitant de cet œcoumène multiple et multicolore est une mer caractéristique, qui présente les traits d'une mer enfermée dans des limites. Nous parlons, évidemment, de la Méditerranée, c'est-à-dire, du véritable berceau d'une multiplicité bien articulée de cultures différentes et en relation réciproque. Cette mer sépare et délimite, mais elle établit aussi une communication entre les côtes qui sont placées l'une en face de l'autre et entre les différentes parties et divers arrière-pays de chacune de ces côtes. Sur cette mer naviguaient pendant les siècles et les millénaires des hommes et des marchandises, des idées et des visions du monde, radicalement différents les uns par rapport aux autres. Les Grecs furent les premiers qui pressentirent l'idée et le projet culturel d'un œcoumène méditerranéen unitaire. Mais ils ne furent pas à même de le réaliser. Seulement les Romains réussirent à donner à cette idée et à ce projet culturel un accomplissement politique très concret, dans le contexte d'un empire maritime et côtier gigantesque. L'univers multiethnique, multireligieux et multiculturel qui fut réalisé par Rome resta toutefois, malgré son extension remarquable, un univers régional et donc fini. 
L'Empire romain maritime et côtier n'était pas encore, à proprement parler, l'Europe.

L'image «biblique» du monde, qui toutefois, du point de vue géohistorique, surgit au cœur de l'œcoumène méditerranéen, est, comme nous l'avons déjà dit, tout autre que l'«homérique». L'image "biblique» du monde, en effet, souligne d'une façon particulière la spatialité maritime, qui montre, en ce cas, des traits fort "océaniques». Cette image est donc placée par Rosenzweig sous le signe de l'unité sans limites et donc de l'infinitude. L'intuition visionnaire des prophètes bibliques est marquée en effet par un élan très puissant visant à rompre toutes les limites géographiques, à surmonter toutes les séparations ethniques et culturelles, à concilier toutes les différentiations. L'inspiration utopique et messianique des prophètes rêve d'une réunification eschatologique de toute la famille humaine sur une terre unique et au-dessous d'un ciel unique, sous le regard d'un Dieu unique, dont il est écrit qu'il a créé l'unique ciel, l'unique terre et l'unique humanité (Rosenzweig 1984, p. 348).

L'intuition biblico-prophétique avait besoin toutefois de conditions historiques et politiques bien concrètes pour commencer à s'accomplir. Ces conditions se produisirent lorsque l'intuition prophétique, accueillie, réinterprétée et répandue par les prêcheurs chrétiens, rencontra une ligne évolutive particulière de la politique expansionniste romaine, une ligne à l'origine tout à fait différente et indépendante par rapport à cette intuition. L'événement historique fondamental qui constitua la prémisse lointaine, d'abord pas du tout évidente, de la rencontre entre l'intuition prophétique et cette ligne évolutive politique et culturelle de la romanité fut représenté, selon Rosenzweig, surtout par la conquête de la Gaule par César. Par cette conquête, un gigantesque tronçon continental - ou, pour ainsi dire, un grand couloir terrestre - mit en communication les deux modalités fondamentales de la spatialité maritime, c'est-à-dire, la méditerranéenne et l'océanique. À l'œkoumène méditerranéen, multiple et culturellement très riche, mais encore seulement régional, fut ouverte de cette façon une porte océanique, qui lui permit de s'élancer audacieusement sur un théâtre inconnu, infini et aux proportions planétaires. Ce fut juste alors que l'idée de l'Europe, avec le projet politique et culturel qui y était relié, commença sa longue marche à travers les siècles et les millénaires. À ce propos Rosenzweig peut écrire: "La conquête de la Gaule par César a été le fondement de l'histoire 
européenne à venir» (Rosenzweig 1984, p. 352). Lidée de l'Europe s'incarna et se dilata dans le projet d'un empire chrétien et universaliste pendant le Moyen Âge, et puis, de différentes façons, toujours plus à l'occasion des grands voyages intercontinentaux de la modernité, et jusqu’à la politique impérialiste des grandes puissances du XIx ${ }^{e}$ siècle. La Première Guerre mondiale représente, selon Rosenzweig, le point d'abordage ultime de cette ligne de développement historique.

Dans ce contexte interprétatif particulier demandons-nous donc: quelle est alors proprement, selon Rosenzweig, l'essence de l'Europe? Entre les lignes de "Globus" se fait jour l'intuition que l'Europe, c'est essentiellement l'idée et le projet politique et culturel d'une universalisation et d'une diffusion planétaires des valeurs spirituelles qui furent élaborées par les cultures méditerranéennes antiques. En ce sens, elle peut donc être, comme nous l'avions déjà anticipé, la rencontre, la liaison et la médiation entre l'image "homérique» et l'image «biblico-prophétique» du monde. S'il m’est permis - précisément à moi, qui ne suis pas français - d'oser deux néologismes, je pourrais dire que l'Europe, dans la perspective du petit ouvrage rosenzweigien, se présente comme une sorte d' "océanisation" singulière de l'image méditerranéenne du monde ou bien, vice versa, comme une "méditerranéisation" tout aussi singulière de l'image océanique du monde. Mais de ce que nous venons de dire découle et apparaît en pleine lumière la vocation dialogique essentielle de l'Europe. L'Europe, c'est la tension à établir des relations signifiantes et productives entre des cultures différentes et parfois même opposées; c'est la création d'un horizon transculturel, à l'intérieur duquel des hommes, des peuples, des langues et des cultures radicalement différents peuvent se parler et se comprendre les uns les autres.

Cette interprétation de l'essence de l'Europe prend toutefois une signification tout à fait particulière si nous nous rappelons les lignes fondamentales de la philosophie de la géohistoire qui est développée dans "Globus». Nous avons déjà eu l'occasion de remarquer en effet que l'image «homérique» du monde souligne d'une façon spéciale la spatialité géographique terrestre, avec les forces motrices historiques, qui y sont reliées, de la séparation, du partage et de la différentiation. Nous avons remarqué aussi que l'image "biblico-prophétique» du monde souligne au contraire la spatialité géographique maritime, avec les forces motrices historiques, qui y sont reliées, de l'intégration et de la réunification entre les différents domaines du monde. Dans cette perspective, 
l'Europe se présente donc comme le projet d'une intégration et d'une synergie - extrêmement créatives - entre le principe de la spatialité terrestre et le principe de la spatialité maritime. L'Europe aurait donc la tâche essentielle d'établir une médiation particulièrement importante et délicate entre la dynamique de la séparation et de la différentiation des domaines du monde et la dynamique de la réunification et de la concordance - «océaniques» et planétaires - de ces domaines mêmes. Cette médiation toutefois, comme nous l'avions déjà dit, représente la direction fondamentale et le but ultime de l'histoire humaine universelle et du dynamisme complexe des forces motrices originaires qui la constituent. Au projet-Europe, par conséquent, Rosenzweig reconnaît un véritable rôle-clef dans l'histoire humaine, qui a été établi par la providence divine.

\section{Le destin de l'Europe}

Dans la dernière partie de mon exposé, je voudrais examiner d'une façon très rapide la méditation rosenzweigienne sur la question du destin ultime de l'Europe telle qu'elle est développée dans "Globus». La question est située par Rosenzweig dans le contexte de la gigantesque transition d'époque qui conduirait l'humanité entière de la scène historique d'avant-guerre, bien connue, mais désormais déclinante, à la scène historique d'après-guerre, qui présentait des traits fort différents. Le point de départ de cette transition est marqué par une scène historique que Rosenzweig pense déjà comme tout à fait planétaire. Dans le contexte de cette scène historique l'Europe, comme entité géopolitique, socioéconomique et culturelle, occupe une place tout à fait centrale. Aux bouts latéraux opposés de cette scène - pour ainsi dire à ses marges - se placent deux mondes socioculturels radicalement différents, c'est-à-dire l'Extrême Orient asiatique et l'Extrême Occident américain. Il me semble particulièrement intéressant de souligner que Rosenzweig, dans sa présentation de l'univers historique d'avant-guerre, utilise un langage métaphorique très précis. Il s'agit en effet du langage de la géométrie plane, qui se meut à l'intérieur d'une spatialité bidimensionnelle. On pourrait dire que la terre n'était pas encore perçue comme ronde par l'humanité d'avant-guerre - tant par les hommes de la rue que par les politiques. Dans ce contexte particulier, cela a certes un sens de parler d'un centre et de bouts latéraux. 
Le résultat fondamental de la Guerre Mondiale, au-delà de ses dimensions militaires et politiques, serait constitué, selon Rosenzweig, par une transformation radicale de la perception et de la représentation de l'espace géographique mondial. L'ancien planisphère bidimensionnel d'avant-guerre aurait dû se courber brusquement sur soi-même en conséquence de la Guerre. Il se serait transformé en une sphère tout à fait tridimensionnelle, c'est-à-dire en ce globe qui constitue justement le titre, la devise et la signification ultime de la réflexion historico-universelle, que Rosenzweig développe dans son petit ouvrage.

La nouvelle scène géopolitique mondiale, qui se serait ouverte à l'Europe et au monde après la fin de la guerre, aurait été marquée par une physionomie fort globale. Et ici il faut attribuer à l'adjectif "global» une signification très prégnante - qui inclut en même temps ses dimensions littérales et métaphoriques. L'événement géopolitique, historique et culturel de la globalisation fut donc perçu par Rosenzweig avec une conscience pleine et aiguë, même si naturellement dans les limites de l'horizon historique et culturel où il se trouvait à son époque. Dans cette scène historique nouvelle d'après-guerre il aurait pu évidemment n'y avoir aucun sens à parler d'un centre du monde, d'une périphérie, de bouts latéraux. Une sphère n'a rien de tout cela sur sa surface. Le monde globalisé aurait donc été un monde où une forme quelconque de séparatisme ou de régionalisme, pensés d'une façon rigoureuse et radicale, serait devenue tout à fait impossible.

À ce point de notre exposé, il est possible d'éclaircir exactement le rôle que l'Europe a joué, selon le penseur, à l'intérieur du processus de globalisation de l'histoire universelle. Il s'agit, en effet d'un rôle de protagoniste absolu. Le monde globalisé peut être interprété alors, de ce point de vue, comme l'accomplissement le plus plein de cette force motrice de l'histoire qui tend à l'universalisation des cultures méditerranéennes et qui constitue, comme nous l'avons déjà dit, l'identité de l'Europe. Nous avons aussi remarqué que cette force motrice de l'histoire a été saisie avec précision par l'intuition "biblico-prophétique» du monde. En cette perspective, le processus de la globalisation peut être interprété comme une politisation surprenante ou comme une sécularisation des intuitions visionnaires - messianiques et utopistes -, qui furent élaborées par le prophétisme biblique et qui furent répandues d'une façon œcuménique par le christianisme. Mais cela signifie que Rosenzweig prête au procès de la globalisation des traits profondément éthiques, solidaires et 
communautaires. Aux yeux de Rosenzweig la globalisation représente un tournant décisif et marquant une date de l'histoire humaine, mais en même temps une chance radicalement positive.

Mais l'intention la plus profonde, qui se cache dans l'arrière-fond de toute la méditation rosenzweigienne sur l'Europe, devient beaucoup plus intelligible si nous relisons, pour ainsi dire, rétrospectivement, "Globus" à la lumière de L'Étoile de la Rédemption. L'un des éléments structuraux les plus remarquables de L'Étoile est représenté, en effet, comme l'on sait, par une polémique vraiment brûlante et parfois même violente contre toutes les formes de totalitarisme, tant politique que philosophique. Cette polémique découle d'une véritable passion pour le thème de la différence, qui, articulé en des façons diverses, joue un rôle tout à fait fondamental dans le chef-d'œuvre du penseur. La polémique contre toute forme de totalitarisme et la passion pour la différence se soudent ensemble très étroitement dans le contexte originel d'une perspective philosophique qui attribue au dialogue une place vraiment prééminente. La globalisation, que Rosenzweig propose comme une chance historique positive, alors, relue à la lumière de sa pensée successive, ne peut - et ne doit - nullement être comprise comme une globalisation qui se produit sous le signe de l'identité et de l'homologation. Elle ne peut pas être une globalisation qui réduit ou qui détruit les différences. La globalisation pour laquelle Rosenzweig se bat est au contraire une globalisation qui exalte et valorise les différences. Il s'agit enfin d'une globalisation des relations interhumaines, une globalisation sous le signe du dialogue et de la solidarité entre les hommes et les peuples.

À l'intérieur de cette perspective l'Europe ne peut plus être le centre du monde. Après la guerre, elle est destinée en effet à perdre irrémédiablement sa prééminence géopolitique, socioéconomique et militaire. "De cette façon", écrit l'auteur, «il semble qu'elle [la guerre] soit du point de vue historico-universel un passage d'une époque européenne passée à une époque planétaire à venir» (Rosenzweig 1984, p. 314). Dans ce nouveau contexte planétaire quelle pourrait être la place de l'Europe et de la culture européenne? Elles seraient appelées, selon Rosenzweig, à devenir l'âme du monde (1984, p. 368). L'âme, certes, ne peut pas du tout être localisée avec précision. Elle remplit au contraire entièrement le corps en lui donnant la vie. Lâme est de plus le principe de la conscience, de la critique, de la moralité, de la pensée. Rosenzweig semble ainsi attribuer à la culture européenne le 
rôle futur, très important, de l'âme d'un monde désormais entièrement globalisé. Il s'agit d'une âme qui, au point de vue historique, a donné autrefois la vie à ce monde. Cette âme pourrait et devrait lui donner encore aujourd'hui un fondement et une signification. Elle peut aspirer à devenir la conscience théorique et éthique, dialogique et interhumaine de ce monde même.

Le message philosophique qui provient de "Globus» a été conçu sans doute dans le contexte d'une époque historique où l'Europe, comme idée et comme projet politique et culturel, semblait se briser irrémédiablement en mille morceaux et où rien ne laissait pressentir le développement de sa réintégration socio-économique et politique qui est devant nos yeux aujourd'hui. La renaissance de l'Europe politique des cendres de deux Guerres mondiales, toutefois, ne suffit pas à nous dispenser d'une réflexion urgente sur les problèmes politiques et culturels que Rosenzweig avait saisis avec une lucidité extrême à son époque. Le contexte globalisé où est en train de s'accomplir l'unité européenne d'aujourd'hui, en particulier, nous impose de repenser à nouveau, de fond en comble, et radicalement, l'identité, le rôle et le destin planétaires de la culture européenne et occidentale. La méditation sur ce sujet qui a été élaborée par le petit officier allemand en 1917 peut bien représenter, à mon avis, encore aujourd'hui un point de départ, d'une actualité renouvelée et surprenante, pour un discours qui est appelé à s'aventurer dans des panoramas impensés.

\section{Bibliographie}

Rosenzweig, F. (1962 [1920]), Hegel und der Staat, 1. Band: Lebensstationen (1770-1806), 2. Band: Weltepochen (1806-1831), Aalen, Scientia.

- (1982), Étoile de la Rédemption, A. Derczanski et J.-L. Schlegel (trads), Paris, Seuil.

-. (1984), Zweistromland. Kleinere Schriften zu Glauben und Denken, R. et A. Mayer (Hrs.), Dordrecht, M. Nijhoff.

—. (1988), Der Stern der Erlösung, Frankfurt am Main, Suhrkamp.

—. (1991), Hegel et l'État, G. Bensussan (trad.), Paris, P.U.F.

-. (2003), Confluences. Politique, histoire, judaïsme, G. Bensussan, M. Crépon et M. de Launay (intr. et trad.), Paris, Vrin.

-. (2007), Globus. Per una teoria storico-universale dello spazio, F. P. Ciglia (a cura di), S. Carretti (trad.), Genova-Milano, Marietti 1820. 